\title{
Advanced imaging capabilities by incorporating plasmonics and metamaterials in detectors
}

John Hennessy, April D. Jewell, Michael E. Hoenk, David Hitlin, Mickel McClish, et al.

John Hennessy, April D. Jewell, Michael E. Hoenk, David Hitlin, Mickel McClish, Alexander G. Carver, Todd J. Jones, Ahmed Morsy, Michelle Povinelli, L. Douglas Bell, Shouleh Nikzad, "Advanced imaging capabilities by incorporating plasmonics and metamaterials in detectors," Proc. SPIE 10639, Micro- and Nanotechnology Sensors, Systems, and Applications X, 106391P (14 May 2018); doi: 10.1117/12.2305126 


\title{
Advanced imaging capabilities by incorporating plasmonics and metamaterials in detectors
}

\author{
John Hennessy $^{\mathrm{a}^{*}}$, April D. Jewell ${ }^{\mathrm{a}}$, Michael E. Hoenk ${ }^{\mathrm{a}}$, David Hitlin ${ }^{\mathrm{b}}$, Mickel McClish ${ }^{\mathrm{c}}$, \\ Alexander G. Carver ${ }^{\mathrm{a}}$, Todd J. Jones ${ }^{\mathrm{a}}$, Ahmed Morsy ${ }^{\mathrm{d}}$, Michelle Povinelli ${ }^{\mathrm{d}}$, L. Douglas Bell ${ }^{\mathrm{a}}$, \\ Shouleh Nikzad ${ }^{\mathrm{a}}$
}

${ }^{a}$ Jet Propulsion Laboratory, California Institute of Technology, 4800 Oak Grove Drive, Pasadena, CA, USA 91109; ' Lauritsen Laboratory, California Institute of Technology, Pasadena, CA, USA

91125; ${ }^{~}$ Radiation Monitoring Devices, Inc., 44 Hunt Street, Watertown, MA, USA 02472; ${ }^{\mathrm{d}}$ Ming Hsieh Department of Electrical Engineering, University of Southern California, 3740 McClintock Avenue, Los Angeles, CA, USA 90089

\begin{abstract}
Ultraviolet detection is often required to be made in the presence of a strong background of solar radiation which needs to be suppressed, but materials limitations at these wavelengths can impact both filter and sensor performance. In this work, we explore the use of 1D photonic bandgap structures integrated directly onto a Si sensor that can operate with solar blindness. These filters take advantage of the improved admittance with silicon to significantly improve throughput over conventional stand-alone bandpass filter elements. At far ultraviolet wavelengths these filters require the use of non-absorbing dielectrics such as the metal fluoride materials of $\mathrm{MgF}_{2}, \mathrm{AlF}_{3}$ and $\mathrm{LiF}$. The latest performance of these 1D multilayer filters on Si photodiodes and CCD imaging sensors is demonstrated. We have also extended these 1D structures to more complex multilayers guided by the design concepts of metamaterials and metatronics, and to 2D patterned plasmonic hole array filters fabricated in aluminum. The performance of sensors and test filter structures is presented with an emphasis on UV throughput.
\end{abstract}

Keywords: ultraviolet, silicon sensor, visible-blind, atomic layer deposition, plasmonics, aluminum

\section{INTRODUCTION}

Materials and device limitations can often restrict imaging and spectroscopy performance at ultraviolet wavelengths. This is compounded by the fact that UV detection is often required to be made in the presence of a strong background of solar or visible radiation, requiring significant filtration if the use of conventional broadband detectors is desired. Backilluminated Si sensors can be fabricated at JPL with a 2D-doping process to yield high quantum efficiency (QE) in the $\mathrm{UV}$, but this high QE extends out to the Si bandgap energy past $1000 \mathrm{~nm}$. We have explored the use of 1D photonic bandgap structures (metal-dielectric multilayers) integrated directly onto a $\mathrm{Si}$ sensor that can maintain high in-band efficiency while providing broad long-wavelength rejection. ${ }^{1}$

Calculated examples of these types of metal-dielectric detector-integrated filters (MDDIF) are shown in Figure 1. Simple three layer structures composed of an aluminum reflector layer surrounded by two dielectric layers can provide a resonant transmission peak of greater than $70 \%$ if deposited directly on the Si surface. This can significantly outperform conventional metal-dielectric filters, particularly at far ultraviolet (FUV, $\lambda=90-200 \mathrm{~nm}$ ) wavelengths where $10-40 \%$ peak transmittances are typical. ${ }^{2}$ It can also outperform simple AR coating efficiencies at FUV wavelengths which are constrained to low-index optical coatings that can provide $50-60 \%$ efficiencies on Si detectors, ${ }^{3}$ but without any long wavelength rejection.

*john.j.hennessy@jpl.nasa.gov

Micro- and Nanotechnology Sensors, Systems, and Applications X, edited by Thomas George,

Achyut K. Dutta, M. Saif Islam, Proc. of SPIE Vol. 10639, 106391P · (c) 2018 SPIE

CCC code: $0277-786 \mathrm{X} / 18 / \$ 18 \cdot$ doi: $10.1117 / 12.2305126$ 


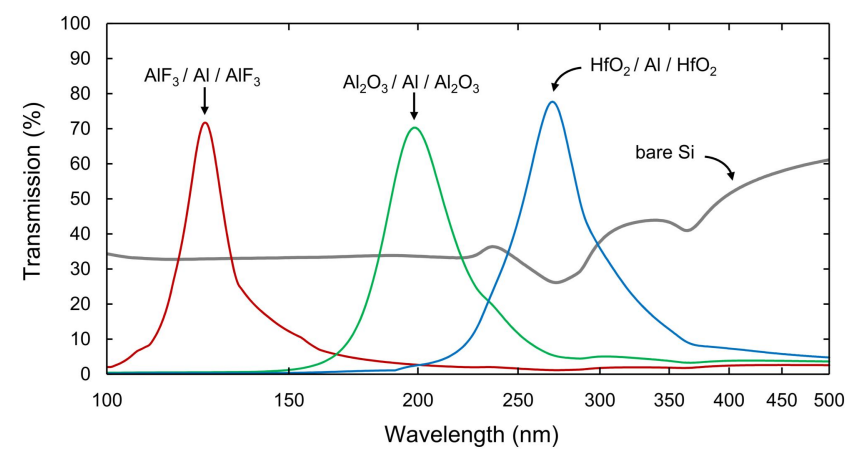

Figure 1. The modeled transmittance of silicon with a native oxide layer in comparison to three-layer metal dielectric filters on silicon using either $\mathrm{HfO}_{2}, \mathrm{Al}_{2} \mathrm{O}_{3}$, or $\mathrm{AlF}_{3}$ as the dielectric spacer and aluminum as the metal. A 0.5 nm oxidation layer on the $\mathrm{Al}$ layer is assumed in each case.

The filters in Figure 1 were optimized to provide near zero reflectance at the target wavelength which may be important for some applications, additional increases in transmission of approximately $10-20 \%$ are possible if the rejection requirements are less critical. The dielectric spacer material can also be varied to provide suppression of shorter wavelength high order passbands, an also to avoid absorption losses when a shorter operating wavelength is required. As discussed in the following section, additional filter cavities can be added to these structures to provide additional rejection or alteration of the bandwidth or spectral shape of the filter.

We have also extended these 1D structures to a greater number of layers with reduced layer thicknesses guided by the design concepts of metamaterials and metatronics. In addition, we have explored extending such 1D multilayer structures to 2D patterned arrays in order to address some of the limitation imposed by a blanket multilayer filter. Nanohole arrays filters fabricated in aluminum via a combination of electron beam lithography and reactive ion etching were analyzed at wavelengths down to $200 \mathrm{~nm}$ on both quartz and Si substrates. The performance of these 2D structures is described in the context of possible future integration with $\mathrm{Si}$ detector systems to enable engineered responsivity to wavelength and polarization in the UV.

\section{MULTILAYER METAL-DIELECTRIC INTEGRATED FILTERS}

The fabrication of MDDIF structures combines evaporated Al layers with the use of atomic layer deposition (ALD) to deposit the transparent spacer layers. For applications at $\lambda>200 \mathrm{~nm}$ many conventional ALD materials like $\mathrm{Al}_{2} \mathrm{O}_{3}$ and $\mathrm{HFO}_{2}$ are suitable for these layers. In the FUV, efficient filter performance is restricted to the use of metal fluoride materials as the spacers. At JPL, we have recently developed several ALD processes for metal fluorides like $\mathrm{MgF}_{2}, \mathrm{AlF}_{3}$, and $\mathrm{LiF}$ using anhydrous $\mathrm{HF}$ as the fluorine-containing precursor. ${ }^{4-6}$ This can theoretically extend the performance of these filters down to the $\mathrm{LiF}$ absorption edge near $105 \mathrm{~nm}$. The use of $\mathrm{AlF}_{3}$ or $\mathrm{MgF}_{2}$, which have slightly smaller band energies, can provide good performance down to $115-120 \mathrm{~nm}$, although other tradeoffs associated with the morphology and environmental stability of the fluoride material may dictate the optimal choice.

We have previously reported on the development of Si avalanche photodiodes (APD) with MDDIFs for use as a readout device to selectively detect the fast scintillation component of $\mathrm{BaF}_{2}$ crystals. ${ }^{7-9}$ The Si APDs undergo a 2D doping process at the wafer-scale in order to improve the UV QE of the back-illuminated device. Our earlier work noted that MDDIF element enforces the most restrictive thermal budget requirement, ${ }^{10}$ therefore in our latest devices this filter step has been moved to the end of the process flow, but is still performed at wafer-scale. The filter coating itself can be patterned and wet-etched to isolate individual devices prior to wafer dicing. Our latest QE results in Figure 2 show improvement over previous demonstrations, and are in good agreement with optical models. The unity-gain QE of these devices is nearly $40 \%$ near the $\mathrm{BaF}_{2}$ scintillation wavelength, with OD3-4 rejection at longer wavelengths. These large area APDs are designed to operate in high gain conditions with the 'effective' QE is predicted to be greater than $50 \%$ according to calculations of the expected width of the depletion region in the device. ${ }^{11}$ 


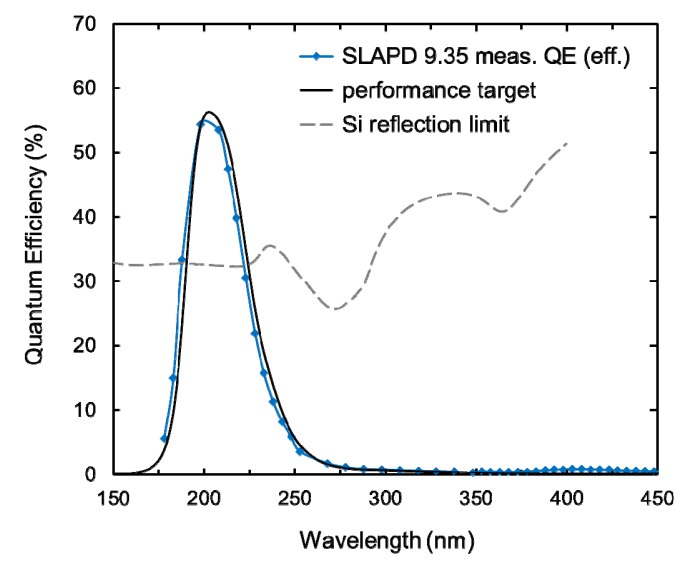

Figure 2. The measured QE of a 2D superlattice doped Si APD with 5 layer MDDIF in comparison the original performance target. The plotted $\mathrm{QE}$ of the device is scale by $1.4 \mathrm{x}$ relative to the raw measurement in order to approximate the effective $\mathrm{QE}$ of the APD at full bias.

\section{INTEGRATION WITH SILICON IMAGING SENSORS AT FUV WAVELENGTHS}

These MDDIFs are also relevant for Si imaging sensor applications. A variety of planetary and astrophysics applications have motivated the demonstration of MDDIFs at FUV wavelengths. ${ }^{11}$ Our work at JPL has focused on demonstrating these structures on back-illuminated CCDs as shown in Figure 3. As mentioned, at $\lambda<200 \mathrm{~nm}$ alternate spacer materials are required to prevent absorption losses in the MDDIF passband. Structures incorporating $\mathrm{Al} / \mathrm{AlF}_{3}$ multilayers have been fabricated on Si CCDs at target wavelengths in the range of $120-175 \mathrm{~nm}$. The use of $\mathrm{AlF}_{3}$ is motivated by its amorphous morphology as-deposited, resulting in reduced surface roughness relative to either $\mathrm{MgF}_{2}$ or $\mathrm{LiF}^{5}{ }^{5} \mathrm{The}$ reduced roughness is expected to have performance benefits as the number of layers in the filter stack is increased. For example, seven layer and nine layer stacks are being consider for similar FUV applications.

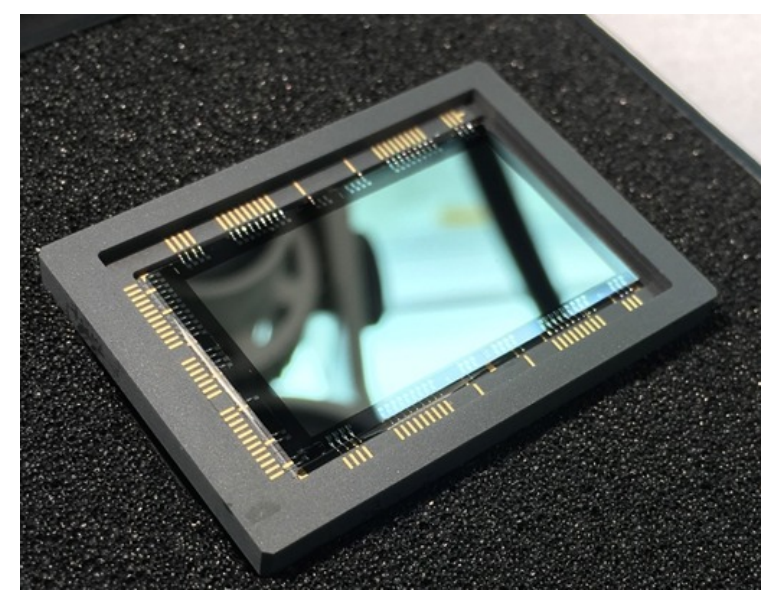

Figure 3. Before and after images of a 2D-doped Si CCD with MDDIF structure of alternating layers of $\mathrm{Al}_{\text {and }} \mathrm{ALD} \mathrm{AlF}_{3}$.

Initial filter performance evaluation has been made by optical characterization of the deposited filters as shown in Figure 4. The measured FUV reflectance is used to fit an optical model of the filter structure in order to predict the filter transmittance in the designed passband. We note that the packaged device pictured in Figure 3 is functional and is capable of imaging with reduced sensitivity to long wavelength light, however calibrated responsivity data was not available at the time of writing. The devices characterized optically in Figure 4 can also be optimized in several ways to 
improve the performance of future iterations. These devices included a thin coating of $\mathrm{ALD} \mathrm{Al}_{2} \mathrm{O}_{3}$ performed at waferscale in order to protect the 2D-doped surface during subsequent processing steps. This layer is lossy at FUV wavelengths and can be eliminated in future iterations to improve passband transmittance by approximately $10 \%$. Another source of loss results from exposing the Al layers to air while they are transferred from the evaporation system to the ALD system. This results in the formation of some finite amount of surface oxide, which would eventually become a performance-limiting factor for MDDIFs with a larger number of cavities. This oxidation is similar to that experience by Al mirror systems which have been investigated with similar ALD processes at JPL. ${ }^{12,13}$ We have explored atomic layer etching (ALE) processes to remove this oxide inside the ALD chamber prior to encapsulation with ALD fluorides, ${ }^{14}$ and initial demonstrations have been promising. ${ }^{15}$ We will incorporate this same ALE method into our MDDIF development to reduce the influence of oxidation on each metal layer in the stack.
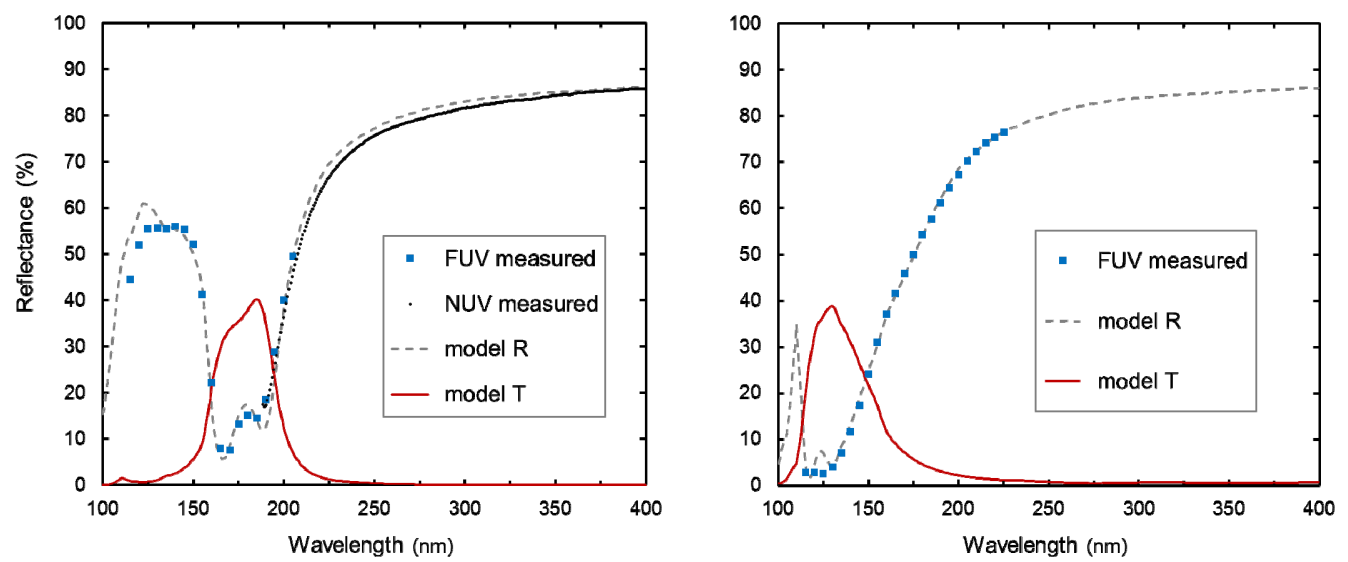

Figure 4. Reflectance characterization of FUV test coatings deposited onto CCDs with a best-fit optical model (dashed line) developed to fit the measured behavior, and the corresponding predicted value of transmittance. In both cases the coating is a five layer stack of $\mathrm{Al} / \mathrm{AlF}_{3}$.

\section{TWO DIMENSIONAL PLASMONIC ARRAYS FOR SILICON INTEGRATION}

The use of 1D MDDIF structures is particularly attractive for spectroscopy applications utilizing Si imaging sensors. In such systems the peak response of the MDDIF could be engineered across the surface of the detector to match the spatial variation of the incident wavelength. In addition to long wavelength rejection, this could also allow for order sorting when multiple octaves of spectrum are desired. Tuning the response in this way would then require spatial variation of the layer thicknesses in the MDDIF. Bottom-up approaches to accomplish this with ALD dielectric layers are challenging due to the non-line-of-sight characteristic of ALD. Top-down patterning and etching is possible, but becomes impractical if large numbers of spectral bands or filter layers are required. This has motivated the investigation of arrays of plasmonic filter structures, and whether the integration of such structures with Si can have similar benefits to the work with MDDIFs. Plasmonics and metasurfaces can be viewed as an engineered versions of these classical optical coatings. Because such structures are much smaller than the pixel in a typical focal plane array, it would then be possible to engineer the response of each pixel in a way that is impractical or impossible with multilayer stacks.

Plasmonic devices take advantage of the coupling of incident light to the collective oscillation of free electrons that can occur in sub-wavelength sized metallic structures. A large amount of work in this field has focused on nanostructures fabricated from gold or silver, which exhibit plasmon resonances from visible to infrared wavelengths; however, at ultraviolet wavelengths these materials exhibit a lossy, non-metallic behavior. Aluminum possesses a higher plasma frequency, and is free of interband transitions through most of the visible and UV, offering a more ideal freeelectron behavior. This leads to a wider spectral tunability range for plasmonic effects in $\mathrm{Al}$, which again permits functionality at UV wavelengths. ${ }^{16}$

The primary structure we have considered are hole array filters ${ }^{17}$ fabricated by ebeam lithography. A variety of structures were consider including structures on glass (stand-alone filter) and also silicon for consideration as an 
integrated filter. The impact of film thickness, lattice period, lattice shape, and hole diameter was studied in this way via finite-difference time-domain simulations. Also considered were hybrid structures that combine conventional 1D continuous films with patterned hole arrays as shown in Figure 5. Calculations suggest such an approach may be a way to tailor the response of these filters in a way that is not possible by simply changing layer thicknesses.

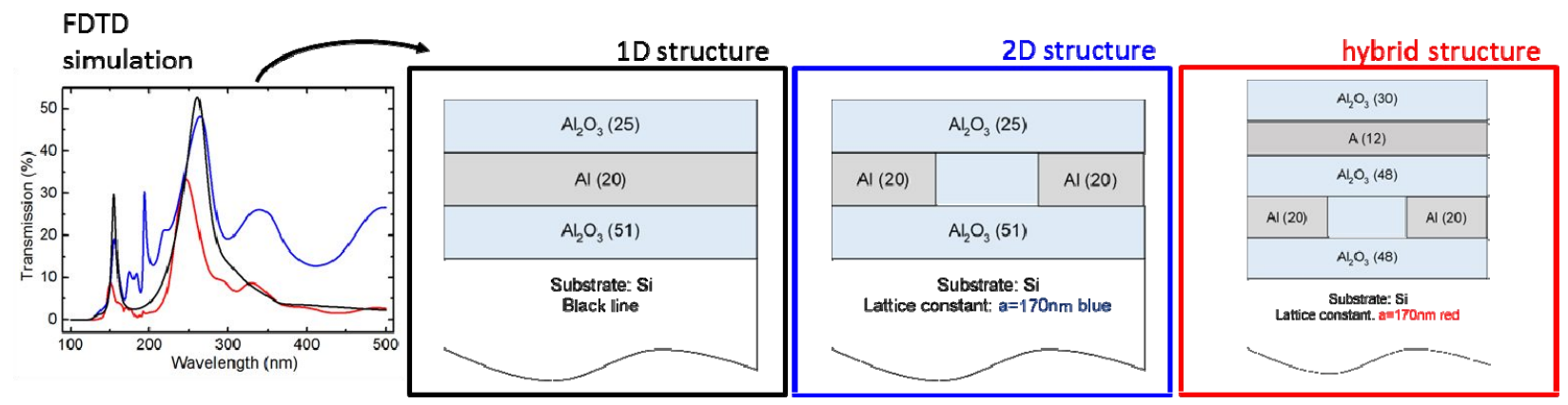

Figure 5. Comparison between the simulated performance of 2D patterned structures to conventional 1D (layered). Hybrid structures may have advantages in throughput or tailoring the shape of bandpass.

A fixed Al thickness of $40 \mathrm{~nm}$ was chosen to constrain analysis and interpretation of the resulting optical performance of hole arrays. Initial samples were fabricated by evaporating Al on UV transparent fused silica substrates. Sixteen patterns were written by ebeam lithography with a variety of array periodicities and hole sizes. The minimum hole diameter as written was approximately $50 \mathrm{~nm}$. The holes were opened via reactive ion plasma etching utilizing test structures to limit overetching the pattern. Similar patterns were subsequently written on equivalent Al films deposited on silicon substrates. ${ }^{18}$ Figure 6 shows a scanning electron micrograph (SEM) of one of these hole array structures. A variety of array structures in square and triangular lattices with varying hole diameter and periodicity have been fabricated and optically characterized.

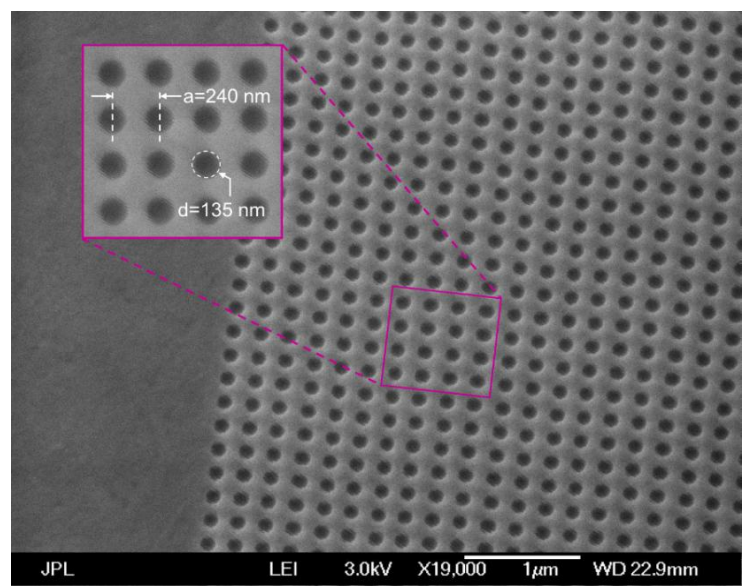

Figure 6. Nanohole arrays fabricated in thin film Al deposited on fused silica, patterned by ebeam lithography and reactive ion etching.

Samples are evaluated in reflection and transmission on a fiber-fed spectrometer illuminated with a xenon lamp. The system is capable of focusing on spot sizes as small as $100 \times 100 \mu \mathrm{m}$, mechanical restrictions for the input and output fibers limit the angle of incidence to a minimum of 35 degrees. Figure 7 show the measured near UV reflectance of a series of test structures with varying periodicity for both square and triangular lattices. These structures are capable of 
achieving resonant features across the near UV that can be tuned with variation of the geometry. In addition to detectorintegrated filters, such structures may have utility in engineering the spatial-spectral response of other optical components like mirrors and polarizers.
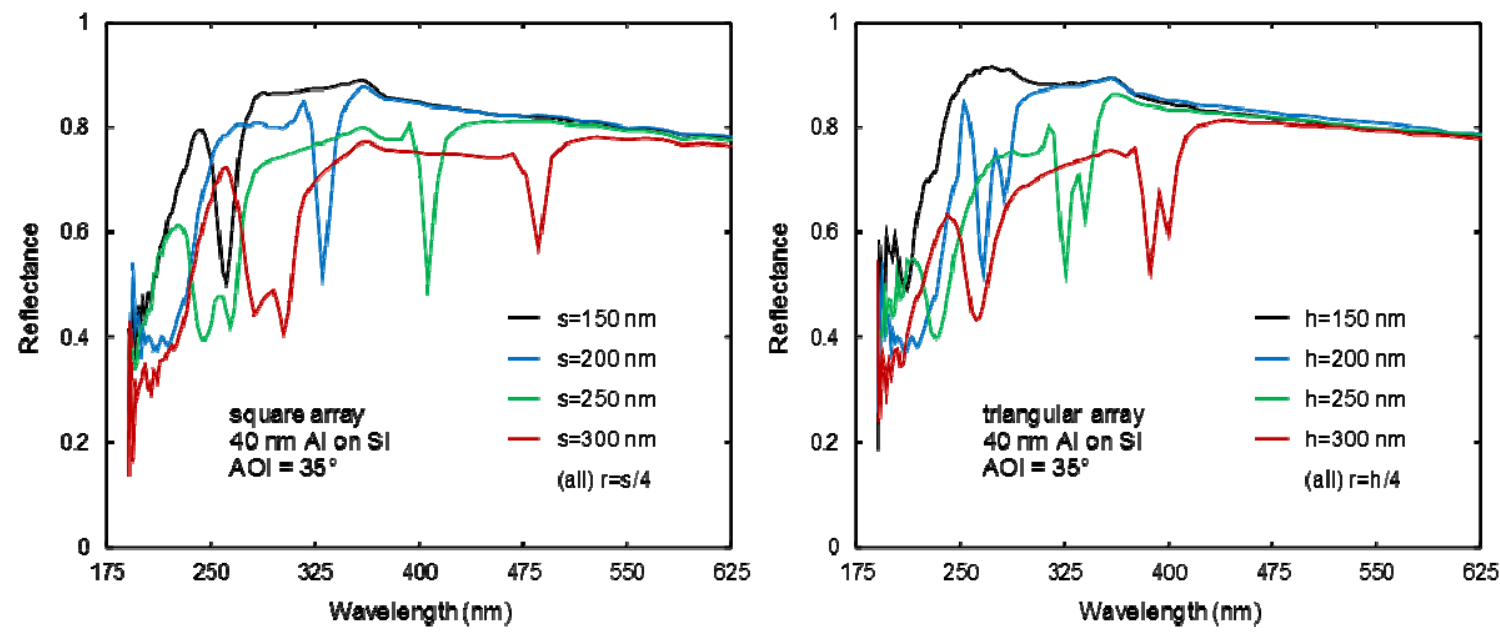

Figure 7. The measured reflectance of patterned hole arrays in aluminum on silicon showing the effect of changing array period for a fixed ratio of hole radius to period for arrays with a square lattice (left) and triangular lattice (right).

\section{ACKNOWLEDGEMENTS}

The research described in this paper was carried out in part at the Jet Propulsion Laboratory, California Institute of Technology, under a contract with the National Aeronautics and Space Administration.

\section{REFERENCES}

[1] Hennessy, J., Jewell, A. D., Hoenk, M. E., and Nikzad, S., "Metal-dielectric filters for solar-blind silicon ultraviolet detectors," Appl. Opt. 54(11), 3507-3512 (2015).

[2] Roming, P. W. A., Kennedy, T. E., Mason, K. O., Nousek, J. A., Ahr, L., Bingham, R. E., Broos, P. S., Carter, M. J., Hancock, B. K., Huckle, H. E., Hunsberger, S. D., Kawakami, H., Killough, R., Koch, T. S., McLelland, M. K., Smith, K., Smith, P. J., Soto, J. C., Boyd, P. T., Breeveld, A. A., Holland, S. T., Ivanushkina, M., Pryzby, M. S., Still, M. D., and Stock, J., "The Swift ultra-violet/optical telescope," Space Sci. Rev. 120, 395-142 (2005).

[3] Nikzad, S., Hoenk, M. E., Greer, F., Jacquot, B., Monacos, S., Jones, T., Blacksberg, J., Hamden, E., Schiminovich, D., Martin, C., and Morrissey, P., "Delta doped electron multiplied CCD with absolute quantum efficiency over $50 \%$ in the near to far ultraviolet range for single photon counting applications," Appl. Opt. 51(3), 365-369 (2012).

[4] Hennessy, J., Jewell, A. D., Greer, F, Lee, M. C., and Nikzad, S., "Atomic layer deposition of magnesium fluoride via bis(ethylcyclopentadienyl)magnesium and anhydrous hydrogen fluoride," J. Vac. Sci. Technol. A 33, 01A125 (2015).

[5] Hennessy, J., Jewell, A. D., Balasubramanian, K., and Nikzad, S., "Ultraviolet optical properties of aluminum fluoride thin films deposited by atomic layer deposition,” J. Vac. Sci. Technol. A 34, 01 A120 (2016).

[6] Hennessy, J., and Nikzad, S., "Atomic Layer Deposition of Lithium Fluoride Optical Coatings for the Ultraviolet," submitted to Inorganics, (2018). 
[7] Hoenk, M. E., Nikzad, S., Carver, A. G., Jones, T. J., Hennessy, J., Jewell, A. D., Sgro, J., Tsur, S., McClish, M., and Farrell, R., "Superlattice-doped silicon detectors: progress and prospects," Proc. SPIE 9154, 915413 (2014).

[8] Hitlin, D., Kim, J. H., Trevor, J., Hennessy, J., Hoenk, M., Jewell, A., Farrell, R. and McClish, M., "An APD for the detection of the fast scintillation component of $\mathrm{BaF}_{2}$," IEEE Trans. Nucl. Sci. 63(2), 513-515 (2016).

[9] Nikzad, S., Hoenk, M., Jewell, A. D., Hennessy, J., Carver, A. G., Jones, T. J., Goodsall, T. M., Hamden, E. T., Suvarna, P., Bulmer, J., and Shahedipour-Sandvik, F., "Single photon counting UV solar-blind detectors using silicon and III-nitride materials," Sensors 16(6), 927 (2016).

[10]Hennessy, J., Jewell, A. D., Hoenk, M. E., Hitlin, D., McClish, M., Carver, A. G., Jones, T. J. and Nikzad, S., "Materials and process development for the fabrication of far ultraviolet device-integrated filters for visible-blind $\mathrm{Si}$ sensors," Proc. SPIE 10209, 102090P (2017).

[11] Nikzad, S., Jewell, A. D., Hoenk, M. E., Jones, T., Hennessy, J., Goodsall, T., Carver, A., Shapiro, C., Cheng, S. R., Hamden, E. and Kyne, G., "High efficiency UV/optical/NIR detectors for large aperture telescopes and UV explorer missions: development of and field observations with delta-doped arrays," J. Astron. Telesc. Instrum. Syst. 3(3), 036002 (2017).

[12] Balasubramanian, K., Hennessy, J., Raouf, N., Nikzad, S., Ayala, M., Shaklan, S., Scowen, P., Del Hoyo, J. and Quijada, M., "Aluminum mirror coatings for UVOIR telescope optics including the far UV," Proc. SPIE 9602, 96020I (2015).

[13]Hennessy, J., Balasubramanian, K., Moore, C. S., Jewell, A. D., Nikzad, S., France, K., and Quijada, M., "Performance and prospects of far ultraviolet aluminum mirrors protected by atomic layer deposition," J. Astron. Telesc. Instrum. Syst. 2(4), 041206 (2016).

[14]Hennessy, J., Moore, C. S., Balasubramanian, K., Jewell, A. D., France, K., and Nikzad, S. "Enhanced atomic layer etching of native aluminum oxide for ultraviolet optical applications," J. Vac. Sci. Technol. A 35, 041512 (2017).

[15] Moore, C.M., Hennessy, J., Balasubramanian, K., Rife, J.C., Vest, R.E., Carter, C., O’Connor, L., Renninger, N., Jewell, A.D., Nikzad, S., and France, K., "Ultrathin protective coatings for enhanced ultraviolet reflectance with aluminum mirrors," submitted to Optics Letters, (2018).

[16] Knight, M. W., King, N. S., Liu, L., Everitt, H. O., Nordlander, P. and Halas, N. J., "Aluminum for plasmonics," ACS Nano 8(1), 834-840 (2013).

[17] Chen, Q. and Cumming, D.R., "High transmission and low color cross-talk plasmonic color filters using triangularlattice hole arrays in aluminum films," Opt. Express 18(13), 14056-14062 (2010).

[18] Morsy, A. M., Povinelli, M. L., and Hennessy, J., "Highly-selective ultraviolet aluminum plasmonic filters on silicon," in preparation (2018). 